\title{
Q OPTIMIZATION VIA QUARTER WAVE ACOUSTIC TRANSFORMERS IN THE BODY OF ALN CONTOUR-MODE RESONATORS
}

\author{
C. Cassella ${ }^{l}$, M. Cremonesi ${ }^{2}$, A. Frangi ${ }^{2}$ and G. Piazza ${ }^{l}$ \\ ${ }^{1}$ Carnegie Mellon University, Pennsylvania, USA \\ ${ }^{2}$ Politecnico of Milan, ITALY
}

\begin{abstract}
This paper investigates the dependence of the quality factor in aluminum nitride contour mode resonators (CMRs) on the size of the inactive region between the resonator body and the lateral edges orthogonal to the main direction of vibration. Our work shows that an optimized length of this region exists and it is such that little energy leaks into the substrate through the supporting anchors. It turns out that the use of the optimum length makes the resonator inactive region behave as an equivalent $\lambda / 4$ transformer. The reduction of the energy that is lost through the device anchors improves the device $Q$ without substantial degradation of the electromechanical coupling, $k_{t}^{2}$. To validate this concept we built four different configurations of $207 \mathrm{MHz}$ AlN CMRs, differing just in the length of the resonator inactive region, $d$ (Fig. 1). An almost $40 \%$ improvement in the Figure of Merit, FoM, defined as the product between $Q$ and $k_{t}^{2}$, was attained when $d$ was varied from the minimum value we tested $(\sim 8 \mu \mathrm{m})$ to the equivalent acoustic quarter wavelength of the resonator inactive region $(\sim 11$ $\mu \mathrm{m})$.
\end{abstract}

\section{INTRODUCTION}

MicroElectroMechanical (MEM) resonators have been widely researched as they enable the implementation of stable frequency references and filtering functions through CMOS compatible fabrication processes. An important parameter of a MEM resonator performance is the quality factor, $Q$, an inverse measure of the energy lost in a passive component. A high $Q$ is highly desirable to ameliorate the performance of both oscillators and filters. However, improving $Q$ in MEM resonators requires a full understanding of the main loss mechanisms as well as the identification of the device parameters that can mitigate their impact.

AlN contour mode resonators (CMRs) have shown low motional resistance and moderate $Q$ in the radio frequency bands. Previous studies have attempted to identify the main dissipation mechanisms affecting this resonator technology. In $[3,5]$ it has been shown that the equivalent damping in AlN CMRs is mostly due to anchor loss and interfacial dissipation. Anchor loss refers to acoustic energy that is lost into the substrate through the supporting anchors. Interfacial dissipation is instead due to stress jumps at the interface between different materials in the resonator's body [2,4]. As shown in [3,5], anchor loss represents the main source of energy dissipation when the device resonance frequency is lower than $500 \mathrm{MHz}$. In contrast, interfacial dissipation $[2,4]$ is the main source of energy lost when the device operates at frequencies greater than $500 \mathrm{MHz}$. Although the impact of the anchor geometry on the device $Q$ was previously investigated [3], there is no understanding of how the length of the resonator inactive region, built between the edge of the active region and the closest stress-free boundary ( $d$ in Fig. 1), affects the device quality factor.

In this work we analyze the variation of $Q$ with respect to $d$ and we verify that it is possible to reduce anchor losses by designing $d$ to be equal to the acoustic quarter wavelength of the resonator inactive region. In fact, in this scenario, each inactive region behaves as a $\lambda / 4$ transformer, hence allowing the resonator active region to see a virtual fixed boundary condition along the anchors direction. As a consequence, the acoustic energy remains confined within the active region and no vibration leaks to the substrate through the supporting anchors.

To validate this new design approach, and to verify an analytical model that describes this phenomenon, four different configurations of $207 \mathrm{MHz}$ AlN CMRs having different lengths, $d$, of the resonator inactive region were analyzed. An ad-hoc developed finite element code was also used to study the device performance as a function of $d$. The use of $\lambda / 4$ transformers in 207 MHz AlN CMRs permitted to attain an almost $40 \%$ improvement of the device $Q$ with respect to what is obtained when $d$ is close to $\lambda / 5$. No substantial variation of $k_{t}^{2}$ with respect to $d$ was measured.

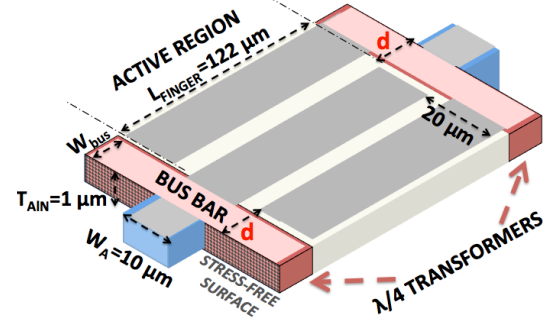

Figure 1: Schematic representation of the $207 \mathrm{MHz}$ AlN CMRs analyzed in this work. Each region included between the edge of the active region and the closest anchor can be designed to act as a $\lambda / 4$ transformer placed in the resonator body.

\section{ALN CONTOUR-MODE RESONATORS}

AlN CMRs are piezoelectric resonators that can excite longitudinal in-plane vibrations in an AIN plate. They are formed by a central active region and two inactive regions (Fig. 1). The active region corresponds to the area in which the electric field generates a mechanical strain through the $\mathrm{AlN} d_{31}$ coefficient. The inactive portions correspond to the areas placed between the edge of the active region and the anchors, where no direct piezoelectric excitation is present.

In this work we have used Lateral Field Excited (LFE) resonators, in which the active region is formed by a thin film of AlN sandwiched between two metal layers that are used to excite the electric field in the AlN film. The top metal layer is an interdigitated structure (IDT), where adjacent metal strips are connected to opposite voltage polarities. The bottom metal layer is a floating metal plate that is used to confine the electric field in the AlN body, hence attaining higher electromechanical coupling, $k_{t}^{2}$.

The inactive region is formed by a sequence of metal covered and uncovered regions and permits to bias each metal strip composing the top metal IDT.

As shown in [1] the resonance frequency of an AIN CMR can be computed as

$$
f=\frac{1}{2 W} \cdot \sqrt{\frac{E_{p}}{\rho_{e q}}}
$$

where $E_{p}$ and $\rho_{e q}$ are respectively the equivalent Young's modulus and mass density of the resonator stack. 


\section{ANCHOR LOSSES}

As shown in [3,5], anchor losses represent the main source of energy lost in AIN CMRs when their resonance frequency is lower than $500 \mathrm{MHz}$. This dissipation mechanism is due to the presence of stress along the anchors direction, at the interface between the supporting anchors and the AlN plate. In particular, when the stress is present at this interface, the anchors are strained, hence transfer energy from the resonator body to the substrate. This phenomenon makes the anchors behave as acoustic waveguides, which limit the maximum quality factor achievable by the device.

Different techniques have been investigated to reduce anchor losses in AlN CMRs. In [3] it has been shown that anchor losses can be reduced when the anchors show an internal resonance in a direction orthogonal to the main direction of propagation of the wave into the substrate. In particular, under these conditions, the flux of energy that would otherwise leak into the substrate is stored into the main mode of vibration, hence reducing the amount of energy lost and, therefore, increasing the resonator $Q$. In [7] it has been shown that anchor dissipations can be further reduced by creating etched slots in the body of AlN CMRs. In this work we show that anchor losses can be minimized by engineering the length of the resonator inactive region ( $d$ in Fig. 1). Although this approach has been validated in AIN CMRs its application can be extended to any similar resonator technology having $Q$ limited by anchor dissipations.

\section{$\lambda / 4$ TRANSFORMERS - PRINCIPLE OF OPERATION}

In this work we show that it is possible to use the acoustic properties of each resonator inactive region (Fig. 1) to limit the amount of energy that is released into the substrate through the device anchors.

Energy dissipation through the anchors is due to longitudinal waves propagating along the anchor direction and being originated by the contour-mode vibration through the AIN Poisson ratio.

To first order, we can express the displacement vector at a generic point, $P$, included in the resonator inactive region, as

$$
\vec{u}_{P}=\int u_{0} \cdot e^{j \vec{k} \cdot \vec{r}} d \vec{r}
$$

where $\vec{k}$ is the wave vector and $\vec{r}$ is a vector connecting each infinitesimal point source forming the edge of the resonator active region to $P$. In Eq. (2) $u_{0}$ is the displacement value at each infinitesimal point source being part of the edge of the active region.

When the anchors include points at which $\vec{u}_{P}$ is non-zero, they let part of the acoustic energy that reaches them leak out of the resonator body, hence limiting the overall device $Q$.

According to Eq. (2) it is straightforward to note that it is possible to limit the amount of anchor dissipations by reducing $u_{0}$. This reduction can be achieved by forcing the edges of the active region to behave as nodal lines. This task can be accomplished by engineering each resonator inactive region to act as $\lambda / 4$ acoustic transformers (Fig. 1). These transformers are capable of imposing virtual fixed boundaries (i.e. $u_{0}$ equal to zero) right at the edge of the resonator active region (Fig. 1-3). Consequently the displacement at the edge of the resonator finger connected to the anchors is also minimized, hence leading to a reduction of the acoustic energy incident on the anchors and, consequently, of the energy lost into the substrate.

For simplicity, assuming initially that the same materials compose both the resonator inactive and active regions, we can write the displacement at the edge of the active region as

$$
u_{0}=u_{i n}-u_{i n} \cdot \Gamma \cdot \cos (2 k d)
$$

where $\Gamma$ is the reflection coefficient computed at the stress-free boundary, hence being equal to -1 , and $d$ is the length of the resonator inactive region. The negative sign in the second term of Eq. (3) is used to identify that the direction of the incident wave is opposite to the wave reflected by the stress-free boundary (i.e. the AlN sidewall).

As there is no energy reflected at the interface between the anchors and the resonator finger connected to them, the energy lost into the substrate is proportional to the product between force and displacement computed at each edge of this finger. Assuming the piezoelectric stress generated in this finger to be almost independent of $d$, the energy lost due to anchor dissipations, $E_{\text {lost }}^{\text {anc }}$, can be written as

$$
E_{\text {lost }}^{a n c} \propto\left(u_{\text {in }}+u_{\text {in }} \cos (2 k d)\right)
$$

This assumption can be adopted as the stress along the anchors direction is generated by the lateral stress in the resonator active region, and hence mostly depends on the resonator stiffness in the lateral direction. The resonator quality factor can then be written as

$$
Q=\frac{E_{\text {stored }}}{E_{\mathrm{var}}+E_{\text {lost }}^{a n c}}=\frac{Q^{\text {eff }}}{\left(1+\frac{E_{\text {lost }}^{a n c}}{E_{\mathrm{var}}}\right)}
$$

where $E_{\text {var }}$ contains the energy that is lost through different dissipation mechanisms and $Q^{e f f}$ is the quality factor that would be achieved if the energy lost through the anchors were zero.

Assuming $E_{v a r}$ to be independent of the dimensions of the resonator inactive region we can express $Q$ as

$$
Q=\frac{Q^{e f f}}{(1+\alpha \cdot(1+\cos (2 k d))}
$$

where $\alpha$ indicates the amount of the resonator energy lost that is related to anchor dissipations. The variation of $Q$, normalized to $Q^{e f f}$, with respect to $d$, for different values of $\alpha$, is shown in Fig.2.

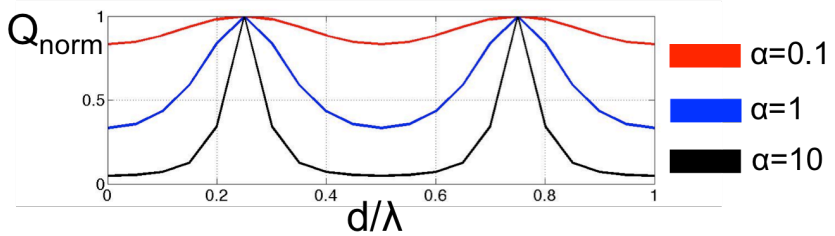

Figure 2: Variation of $Q$, normalized to $Q^{e f f}(E q .6)$, with respect to the ratio between the length of the resonator inactive region and the acoustic wavelength, for different values of $\alpha$. The trend suggests that the bandwidth of each $\lambda / 4$ transformer is inversely proportional to the value of $\alpha$. No variation of the acoustic velocity between transformer and resonator active region is considered here.

As evident from Eq. (6), and as intuitively expected, the variation of the quality factor with respect to the distance, $d$, is strongly influenced by $\alpha$. The higher the impact of anchor losses on $Q$, the greater the relevance of the size of $d$ in setting the overall device $Q$. However the maximum quality factor is always achieved when $d$ is equal to odd multiples of $\lambda / 4$. This scenario corresponds to the case in which the edges of the active region are virtually clamped along the anchor direction. 


\section{$\lambda / 4$ TRANSFORMERS - DESIGN CONSIDERATION}

We analyzed the variation of $Q$ with respect to the length of the resonator inactive region in 3-finger $207 \mathrm{MHz}$ AlN CMRs. This analysis was done by varying the width of the two metal strips (one on each side of the resonator) that are used to connect the electrodes forming the top IDT to the proper voltage polarity (Fig. 1-3). This width, labeled as $W_{b u s}$, is equivalent to $d-\lambda / 10, \lambda$ being equal to $40 \mu \mathrm{m}$ at the frequency of interest. As in the actual design the resonator inactive region is formed by multiple sections (Fig. 3), each having a different acoustic velocity, we recurred to the transmission line theory to find the value of $W_{b u s}$ that maximizes the device $Q$. Each section forming the resonator inactive region was modeled as a transmission line having length equal to its actual size and characteristic impedance and propagation constant set by its acoustic property.

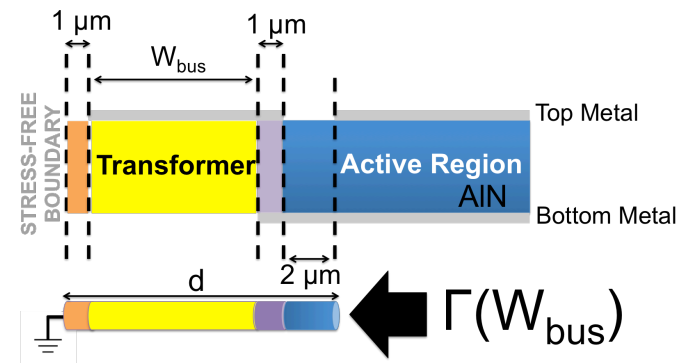

Figure 3: Cross-sectional schematic of the transformer under study. Each transformer is formed by four different sections characterized by different acoustic velocities. Both metal layers are made by platinum. Their thickness is about $0.1 \mu \mathrm{m}$. Both the active region and the transformers use the same AlN film having a thickness equal to $1 \mu \mathrm{m}$.

By looking at the reflection coefficient, $\Gamma$ (Fig.1-3), defined as the ratio between incident and reflected waves at the edge of the active region, we found the value of $W_{b u s}$ that allows transforming the stress-free boundary into a fixed boundary. This value, $W_{b u s}{ }^{o p t}$, corresponds to the size of the bus that makes the real part of $\Gamma$ (Fig. 3 ) equal to 1 (Fig. 4). It is important to notice that the change in phase of the reflection coefficient observed by the lateral fingers (Fig. 1) is needed as it permits to virtually clamp the edge of the central finger connected to the anchor.

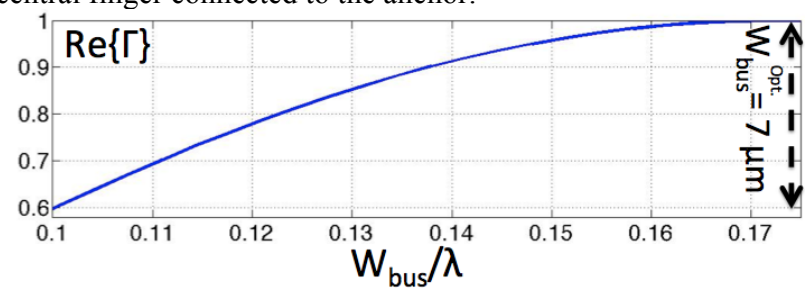

Figure 4: Real part of the reflection coefficient $\Gamma$ as defined in Fig. 1 and Fig. 3. The presence of different sections in each transformer increases the size of the resonator inactive region that corresponds to $\lambda / 4$ by a factor close to $10 \%$ with respect to the scenario in which the same acoustic velocity is assumed for both the resonator active and inactive regions.

\section{DEVICE FABRICATION}

In Fig. 5 we show the fabrication process we followed to build the devices we analyzed in this work. We first deposited $100 \mathrm{~nm}$ of platinum above $10 \mathrm{~nm}$ of titanium (Fig. 5-a). Afterwards we deposited $1 \mu \mathrm{m}$ of AlN (Fig. 5-b) followed by the deposition of $100 \mathrm{~nm}$ of platinum (Fig. 5-c) top electrode. The etching of the AlN (Fig. 5-d), followed by the structural release (Fig. 7-e), completed the device fabrication.

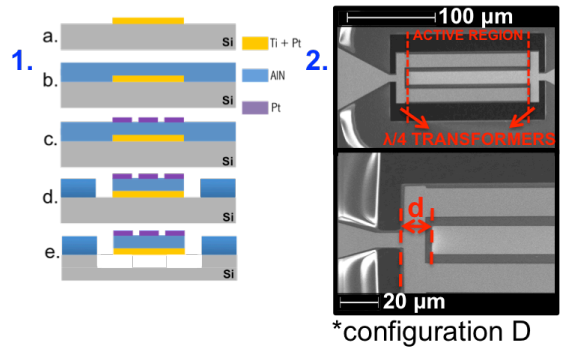

Figure 5: 1. Fabrication process for the AlN resonators built for this work: 2. SEM pictures of a tested device and zoomed in view of the transformer region.

\section{EXPERIMENTAL RESULTS}

To evaluate the variation of $Q$ when $W_{\text {bus }}$ approaches $W_{\text {bus }}^{\text {opt }}$, we built four different configurations of $207 \mathrm{MHz}$ AlN CMRs differing just for the value of $W_{b u s}$ (Fig. 3). These 4 resonators were built with the optimum anchor width, $W_{A}=10 \mu \mathrm{m}$ (Fig. 1), which was found by measuring 36 resonators having $W_{A}$ ranging from 10 to $60 \mu \mathrm{m}$.

$W_{\text {bus }}$ was chosen to be $4,5,6$ and $7 \mu \mathrm{m}$ respectively in configurations $\mathrm{A}, \mathrm{B}, \mathrm{C}$ and $\mathrm{D}$. We tested 6 devices for configuration A, 12 devices for configuration $\mathrm{B}, 6$ devices for configuration $\mathrm{C}$, and 6 devices for configuration $\mathrm{D}$. All the devices were extracted from 3 chips belonging to the same wafer.

We report in Fig. 6 the average $Q$ we measured for each configuration. Moreover, by using Eq. (6), in which $Q^{\text {eff }}$ was set to be equal to the maximum average $Q$ we measured, we were able to match closely the measured variation of $Q$ with respect to $W_{b u s}$ by using a coefficient $\alpha$ equal to 1.5. The fact that the value of $\alpha$ that permits to match the experimental results is greater than one shows that anchor dissipations are greater than any other loss mechanism in $207 \mathrm{MHz}$ AlN CMRs. However, this fact also proves that different dissipation mechanisms contribute significantly in limiting the quality factor achievable by this resonator technology operating in the Very High Frequency (VHF) range. It is important to clarify that, although Eq. (6) was formulated assuming that the resonator active region was formed by the same material than the transformers, it still can be used when the transformer topology is the one depicted in Fig. 3. This is possible since the magnitude of the reflection coefficient observed by the central finger looking at the anchors is approximately $-30 \mathrm{~dB}$ and almost independent of $W_{\text {bus. }}$.

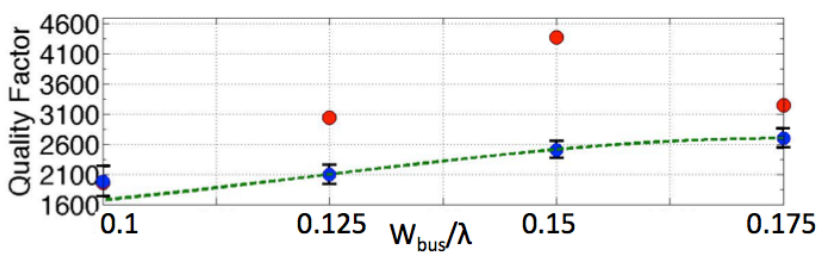

Figure 6: In blue: Averaged quality factor, $Q$, measured in configurations $A, B, C$ and $D$. About $40 \%$ improvement of $Q$ was measured going from configuration $A\left(W_{\text {bus }}=4 \mu \mathrm{m}\right)$ to configuration $D\left(W_{\text {bus }}=7 \mu \mathrm{m}\right)$ In red: Simulated $Q$ by FEM analysis. The presence of metal covered areas in the inactive region was neglected. Because of this assumption the maximum $Q$ is simulated at $d$ equal to the equivalent resonator quarter-wave length. In green: analytical estimation of the resonator $Q$ as a function of the ratio $W_{\text {bus }} / \lambda$. We speculate that the discrepancy between the analytical trend and the measured $Q$ at $W_{\text {bus }}$ equal to 4 $\mu \mathrm{m}$ is due to the presence of a spurious mode in the resonator inactive region that changes its transmission properties. 
In Fig. 7 we report the best admittance we measured for each configuration we tested. Passing from configuration $\mathrm{A}$ to configuration D we observed a progressive slight reduction of $k_{t}^{2}$ motivated by the increased static capacitance due to the increase of $W_{b u s}$. Moreover a reduction of the resonance frequency was also measured as $W_{b u s}$ was increased.

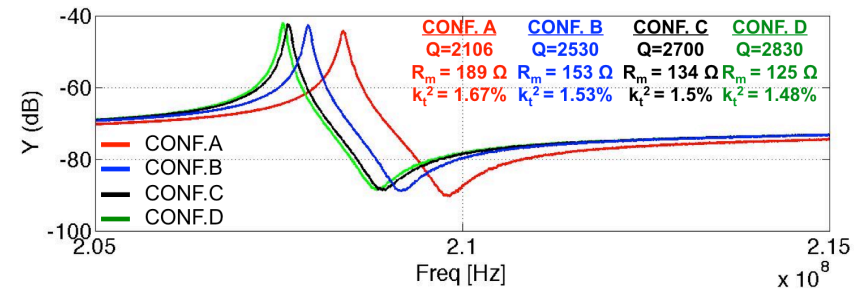

Figure 7: Best-measured admittance response for the four configurations analyzed in this work. Values of the motional resistance, $R_{m}$, and $k_{t}^{2}$ are also reported.

This fact is due to the larger value of the resonator effective mass as $W_{b u s}$ is increased. However the fact that the sensitivity of the resonance frequency with respect to $W_{b u s}$ decreases when $W_{b u s}$ approaches $W_{\text {bus }}{ }^{\text {opt }}$ represents a further indication that the displacement in the resonator inactive region, and consequently in the anchors, approaches zero in configuration D.

\section{FEM ANALYSIS}

In order to obtain a quantitative prediction of the overall quality factor, we assume the presence of both anchor and interface losses [3-7]. Anchor losses have been estimated by means of a robust Finite Element Method including absorbing boundary regions (PML) and simulating the dissipation of elastic waves scattered in the substrate through the anchors. Exploiting the symmetry of the devices, only 1/4-th of the resonators is discretized. Fig. 8 shows the geometrical model and the contour plot of the displacement field corresponding to the mechanical mode actuated.

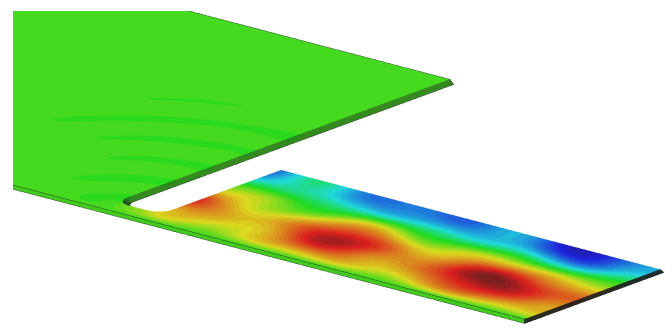

Figure 8: Model of $1 / 4^{\text {th }}$ of the resonator. Contour plot of the displacement field corresponding to the mechanical mode actuated.

On the contrary, Fig. 9 presents the pattern of scattered elastic waves superposed to the finite element mesh adopted in the simulations; their smooth dissipation in the PML region validates the accuracy of the numerical model. The complex eigenfrequency $\Omega_{0}^{2}$, corresponding to the activated mechanical mode, is computed and the quality factor due to anchor losses is estimated as:

$$
Q_{\text {anc }}=\operatorname{Re}\left(\Omega_{0}^{2}\right) / \operatorname{Im}\left(\Omega_{0}^{2}\right)
$$

Interface losses are described employing a phenomenological law implemented in the FEM model. Assuming a 1D extensional mode of vibration, it has been recently proposed that interface losses might be associated with the Cauchy stress jump $\Delta \sigma$ across the interfaces between different materials:

$$
\Delta \sigma=\Delta E \frac{\partial u}{\partial x}
$$

where $u$ corresponds to the lateral acoustic displacement and $\Delta E$ is the discontinuity of Young modulus.

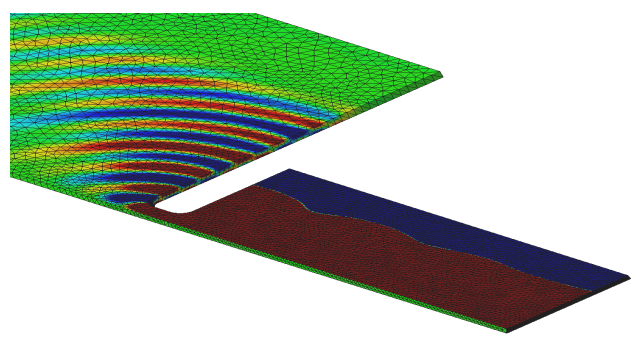

Figure 9: Pattern of scattered elastic waves and final dissipation in the PML region.

As previously formulated in [3-4,6], we postulate that the energy lost over one cycle $T$ due to interfacial dissipation $\left(E_{\text {int }}\right)$ at each interface $S$ of the mechanical resonators is:

$$
E_{\text {int }}=\eta \int_{S} \int_{0}^{T}(\Delta \dot{\sigma})^{2} d t d S
$$

where $\eta$ is a material parameter.

The numerical results collected in Figure 6 reveal a strong sensitivity of the quality factor to $W_{\text {bus }}$ and a good qualitative agreement with experimental data. It is worth stressing that, at present, simulations of anchor losses employ a homogenized resonator and do not represent the details of metallic interdigitated fingers. Since anchor losses are strongly sensitive to all the geometrical and material parameters, a more accurate model is expected to improve the predictive capabilities.

\section{CONCLUSIONS}

In this work, we provide theoretical and experimental demonstration of the dependence of the quality factor of AlN contour-mode resonators (CMRs) on the size of the resonator inactive region. We found that the optimization of this region permits to limit the amount of acoustic energy that leaks into the substrate through the device anchors and, consequently, maximizes Q. A $40 \%$ increase in the quality factor of $207 \mathrm{MHz}$ AlN CMRs was measured when the size of the resonator inactive region was varied from $\lambda / 5$ to $\lambda / 4$, being $\lambda$ the acoustic wavelength. This work also demonstrates that anchor loss is not the only significant dissipation mechanism that affects the quality factor in AIN CMRs operating in the Very High Frequency (VHF) range.

\section{REFERENCES}

[1] G. Piazza, P. J. Stephanou, A. P. Pisano, Journal of MEMS,vol. 15, no. 6, pp. 1406-1418, 2006.

[2] L.-W. Hung and C. T.-C. Nguyen, Solid-State Sensor and Actuator Workshop, pp. 463-466, 2010.

[3] J.Segovia, M.Cremonesi, C.Cassella, A.Frangi, G.Piazza, In SolidState Sensors, Actuators and Microsystems, 2013 Transducers \& Eurosensors XXVII: The 17th International Conference on (pp. 24732476). IEEE.

[4] C.Cassella, J.Segovia-Fernandez, G.Piazza, In Solid-State Sensors, Actuators and Microsystems, 2013 Transducers \& Eurosensors XXVII: The 17th International Conference on (pp. 506-509). IEEE.

[5] A. Frangi, M. Cremonesi, A. Jaakkola, T. Pensala, Sensors \& Actuators: A Physical, vol. 190, pp. 137-134, 2013.

[6] A. Frangi, A. Bugada, M. Martelo, P.T. Savadkoohi, European Journal of Mechanics - A/Solids, vol. 37, pp. 256-265, 2013.

[7] Cassella, C., Segovia-Fernandez, J., Piazza, G., Cremonesi, M., \& Frangi, A., In Frequency Control Symposium (EFTF/IFC), 2013 Joint (pp. 926-929). IEEE. 